\title{
Simulation of single bubble rising in liquid using front tracking method
}

\author{
J. Hua \& J. Lou \\ Institute of High Performance Computing, \#01-01 The Capricorn, \\ Singapore
}

\begin{abstract}
Front tracking method is improved to simulate the rising and deforming of a bubble in quiescent viscous liquid under various flow regimes. The simulation results demonstrate that the current algorithm is more robust in modelling a multi-fluid system with wider ranges of the Reynolds number $(1<\operatorname{Re}<200)$ and the Bond number $(1<$ Bo $<200)$ under a high density ratio $\left(\rho_{1} / \rho_{b}=1000\right)$ and a viscosity ratio $\left(\mu_{1} / \mu_{b}=100\right)$. The new front tracking algorithm is also applied to investigate bubble rising and deforming behaviour in the various flow regimes of the "air bubble-water solution" system under effects of the Reynolds number and the Bond number, which have been explored experimentally in literature. The simulation results are compared with the experiments in aspects of bubble shape, terminal velocity and bubble wake flow pattern. Reasonable agreements are achieved in most flow regimes.
\end{abstract}

Keywords: bubble rising, front tracking method, numerical simulation, multiphase flow.

\section{Introduction}

Multi-fluid systems play an important role in many natural and industrial processes such as combustion / chemical reacting, petroleum refining, boiling, etc. The rising of single bubble driven by buoyancy force in viscous liquid is one of such typical multi-fluid systems. A comprehensive understanding of the flow behaviour and mechanism of such multi-fluid systems in full flow regimes has not been well developed so far.

In the present work, front tracking method, a hybrid approach of front capturing and tracking technique proposed by Unverdi and Tryggvason [1] for 
modelling multi-fluid system was examined. In this method, a stationary, fixed grid is used for the fluid flow, and a set of adaptive elements on the front is used to represent the interface. A single set of governing equations (Navier-Stokes equation and continuity equation) for the whole computational domain is solved for the two phases by treating the different phases as one fluid with variable material properties. The fluid properties such as density and viscosity are calculated based on the position of the interface, and updated basing on the motion of the bubble front. Hence, this method could avoid numerical diffusion, and capture sharp interfaces. Interfacial source terms such as surface tension are computed on the front and transferred to the fixed background grid using a $\delta$ (dirac-delta) distribution function from the interface between the phases. The front tracking method has been applied to various interfacial flow problems [1]. It has been noted that the capability of conventional algorithm is limited to lower density ratios, lower Reynolds numbers and lower Bond numbers [2]. A further extension of this method is proposed in this paper to make it applicable to wider flow regimes.

As benchmarking tests, the new algorithm is used to model single air bubble rising in viscous liquid, which actually has been treated as typical cases to validate numerical methods for multiphase/interfacial flows. Most of previous validation works are performed under conditions of lower density ratio, the simulation results are validated against experimental observations about bubble shape under some typical flow regimes [3, 4]. In this paper, the front tracking method is used to systematically investigate air bubble rising in water solutions under various flow regimes. The simulation results are compared with experimental results in bubble shape, terminal velocity and wake flow pattern, and good agreement can be obtained in different flow regimes.

\section{Mathematical formulation and numerical method}

\subsection{Governing equations}

In this study, we investigate the rise of a bubble in a quiescent liquid. It is normally reasonable to treat both liquid and bubble phases as incompressible fluid. Hence, the mass conservation equation on the whole domain (both fluid phases and the interface) may be expressed as,

$$
\nabla \cdot \mathbf{u}=0
$$

The Navier-Stokes equation, governing the momentum balance in each fluid domain and the interface, may be expressed as,

$$
\frac{\partial(\rho \mathbf{u})}{\partial t}+\nabla \cdot \rho \mathbf{u u}=-\nabla p+\nabla \cdot\left[\mu\left(\nabla \mathbf{u}+\nabla^{T} \mathbf{u}\right)\right]+\sigma \kappa \mathbf{n} \delta\left(\mathbf{x}-\mathbf{x}_{f}\right)+\left(\rho-\rho_{l}\right) \mathbf{g}
$$

where, $p$ is the pressure in the fluid domain, $\sigma$ is the surface tension, $\kappa$ is the curvature of the interface. $\delta\left(\mathbf{x}-\mathbf{x}_{f}\right)$ is a delta function that is zero everywhere except at the interface, i.e., at $\mathbf{x}=\mathbf{x}_{f} \cdot \mathbf{g}$ is the gravitational acceleration, and 
subscript $f$ refers the front / interface. $\rho$ refers the density of fluid, and $\rho_{l}$ the density of liquid phase.

We introduce the following dimensionless characteristic variables,

$$
x^{*}=\frac{x}{D} ; u^{*}=\frac{u}{(g D)^{1 / 2}} ; \tau^{*}=\frac{t}{D^{1 / 2} g^{-1 / 2}} ; \rho^{*}=\frac{\rho}{\rho_{l}} ; p^{*}=\frac{p}{\rho_{l} g D} ; \mu^{*}=\frac{\mu}{\mu_{l}} ; \kappa^{*}=\frac{\kappa}{D^{-1}} \text {. }
$$

where $\mathrm{D}$ is the effective diameter of a bubble and defined as $D=\left(6 V_{b} / \pi\right)^{1 / 3}$ and $V_{b}$ is the bubble volume. The subscripts $l$ and $b$ stand for the liquid and gas bubble phases, respectively. So, the non-dimensional Navier-Stokes equation may be re-expressed as,

$$
\frac{\partial(\rho \mathbf{u})}{\partial t}+\nabla \cdot \rho \mathbf{u} \mathbf{u}=-\nabla p+\frac{1}{\operatorname{Re}} \nabla \cdot\left[\mu\left(\nabla \mathbf{u}+\nabla^{T} \mathbf{u}\right)\right]+\frac{1}{\mathrm{Bo}} \kappa \mathbf{n} \delta\left(\mathbf{x}-\mathbf{x}_{f}\right)+(\rho-1) \mathbf{g},
$$

in which the superscript $*$ is omitted for convenience, while the non-dimensional Reynolds number, Bond number (also known as Eotvos number) and Morton number are defined as following,

$$
\operatorname{Re}^{*}=\frac{\rho_{l} g^{1 / 2} D^{3 / 2}}{\mu_{l}} ; \mathrm{Bo}^{*}=\frac{\rho_{l} g D^{2}}{\sigma} ;
$$

In the experimental work [5], the following dimensionless parameters, Morton (M) and Reynolds numbers (Re), are also used to characterise the fluid flow,

$$
\operatorname{Re}=\frac{\rho_{l} D U_{\infty}}{\mu_{l}} ; \mathrm{M}=\frac{g \mu_{l}^{4}}{\rho_{l} \sigma^{3}}=\frac{\mathrm{Bo}^{3}}{\mathrm{Re}^{4}} .
$$

\subsection{Treatment of the discontinuities across the front}

The novelty of the front tacking method proposed by Unverdi and Tryggvason [1] is that the front is considered to have a finite thickness of the order of the mesh size instead of zero thickness. In the transition zone around the interface, the fluid properties change smoothly and continuously from the value on one side of the interface to the value on the other side. The material property fields over whole domain may be reconstructed using an indicator function $I(\mathbf{x}, t)$, which has the value of one in the bubble gas phase and zero in the liquid phase at a given time $t$.

$$
b(\mathbf{x}, t)=b_{l}+\left(b_{b}-b_{l}\right) I(\mathbf{x}, t) ; \quad I(\mathbf{x}, t)=\int_{\Omega(t)} \delta\left(\mathbf{x}-\mathbf{x}^{\prime}\right) d \mathbf{v}^{\prime}
$$

in which $b$ stands for either fluid density or viscosity. The indicator function can be written in the form of an integral over the whole domain $\Omega(t)$ bounded by the phase interface $\Gamma(t) . \delta\left(\mathbf{x}-\mathbf{x}^{\prime}\right)$ is a delta function that has a value of one where $\mathbf{x}^{\prime}=\mathbf{x}$ and zero everywhere else. In this study, the delta function is 
approximated by the following distribution function $D(\mathbf{x})$ suggested by Peskin [6] are used for a two-dimensional grid system,

$$
D\left(\mathbf{x}-\mathbf{x}_{f}\right)=\left\{\begin{array}{cl}
(4 h)^{-2} \prod_{i=1}^{2}\left(1+\cos \left(\frac{\pi}{2 h}\left|\mathbf{x}-\mathbf{x}_{f}\right|\right)\right), & \text { if }\left|\mathbf{x}-\mathbf{x}_{f}\right|<2 \mathrm{~h} \\
0, & \text { otherwise. }
\end{array}\right.
$$

where $h$ is the background mesh grid size. The distribution function defines the fraction of the interface quantity can be distributed to nearby grid point across the artificial thickness of the front.

\subsection{Numerical method}

Projection method for the integration of the Navier-Stokes equations (3) was used in the previous works of Unverdi and Tryggvason [1]. The difficulties in solving the above pressure equation have been reported. For example, a large density ratio may lead to a problem in convergence $[1,2]$. In order to overcome the difficulties in solving the pressure equation, an alternative approach is implemented in the present work. Here, the coupling fluid velocity and pressure is updated by solving the momentum equations and continuity equation using SIMPLE scheme [7], and simulation process is more robust even in case of large density ratio because of the semi-implicit solving approach. In the multi-fluid system, due to density jump over the phase interface, mass flux conservation in the control volume crossing the front interface is not valid. Hence, volume flux conservation is adopted to modify the SIMPLE algorithm. Based on this approach, SIMPLE algorithm is used to calculate the correction value of pressure and velocity after solving the momentum equation.

\subsection{Front tracking}

Since the fluid velocity is updated on the fixed grid, the node moving velocity on the front should be computed by interpolating from the fixed grid to ensure that the front moves at the same velocity as the surrounding fluids. In section 2.2 , the distribution function used to spread the fluid property jump to the fixed points nearby the interface was discussed. Similarly, this function can also be used to interpolate field variables from the fixed background grid to the front using the following equation,

$$
\mathbf{u}_{f}=\sum D\left(\mathbf{x}_{f}-\mathbf{x}\right) \mathbf{u}(\mathbf{x})
$$

Then, the front is advected along its normal direction in a Lagrangian fashion,

$$
\mathbf{x}_{f}{ }^{n+1}-\mathbf{x}_{f}{ }^{n}=\Delta t \mathbf{u}_{f} \cdot \mathbf{n}
$$

After the position of the front is updated, the front elements should be adapted to maintain the element quality. 


\subsection{Solution procedure}

With appropriate initial conditions for the fluid flow and interface shape, the solution algorithm proceeds iteratively through the following steps:

(1) Using the fluid velocity field $\left(\mathbf{u}^{n}\right)$ and the interface position $\left(\mathbf{x}_{f}{ }^{n}\right)$, the moving velocity of the front marker points $\left(\mathbf{u}_{f}{ }^{n}\right)$ is computed using equation (6).

(2) Using the estimated normal interface velocity, the front is advected to the new position $\left(\mathbf{x}_{f}{ }^{n+1}\right)$. Subsequently, the elements, representing the front, are examined for adaptation and topology change.

(3) At the new interface positions, the redistribution of the interface property is performed with the reconstructed indicator function $I\left(\mathbf{x}_{f}{ }^{n+1}\right)$. Hence, new fluid property filed such as density $\left(\rho^{n+1}\right)$, viscosity $\left(\mu^{n+1}\right)$, as well as the surface tension $\left(\mathbf{F}_{s t}{ }^{n+1}\right)$, are obtained

(4) With appropriate wall boundary conditions, the momentum equation and mass continuity equation can be solved using the modified SIMPLE algorithm. This leads to update fluid velocity $\left(\mathbf{u}^{n+1}\right)$ and pressure $\left(p^{n+1}\right)$.

(5) Repeat the solution steps from (1) to (4) for the next time step calculation.

\section{Results and discussion}

\subsection{Experimental observation of terminal bubble shape and rising velocity}

Experimental studies on the rise of single bubble in quiescent liquid have been reported in the literature $[5,8]$. The terminal bubble shapes vary greatly in different flow regimes as a function of the non-dimensional parameters such as Eotvos number (Bond number), Reynolds number and Morton number. The terminal shapes of single bubble rising in quiescent liquid under a range of Reynolds and Bound numbers $(\operatorname{Re}<200, \mathrm{Bo}<200)$ were shown in Figure 1. In general, the rising bubbles have axisymmetric shapes when the Reynolds and Bond numbers are not too higher $(\operatorname{Re}<200, \mathrm{Bo}<200)$. Hence, the single bubble rising in quiescent liquid under such flow regimes, which produce axisymmetric bubbles, could be simulated using the front tracking method in an axisymmetric co-ordinate system.

\subsection{The effect of Reynolds and Bond numbers on the bubble shape}

The simulation predicted bubble shapes in a wide range of Reynolds and Bond numbers are summarised in Figure 2. In the regimes of low Reynolds and Bond numbers $\left(\operatorname{Re}^{*}<1\right.$ and $\left.\mathrm{Bo}^{*}<5\right)$, the bubbles remain spherical while they are rising in the liquid. With a slight increase in Reynolds number $\left(\operatorname{Re}^{*}=10\right)$, the bubble shape still remains spherical for low Bond number, where surface tension is higher. On the other end of high Bond numbers, the bubble bottom becomes flat or slightly dimpled. With the further increases in Reynolds number $\left(10<\operatorname{Re}^{*}<20\right)$ and Bond number $\left(5<\mathrm{Bo}^{*}<20\right)$, bubbles in the shapes of 
elliptic/oblate ellipsoid are observed. With further increase in Reynolds number $\left(100<\operatorname{Re}^{*}<200\right)$ and Bond number $\left(20<\mathrm{Bo}^{*}<100\right)$, the bubble shapes range from highly deformed elliptical-cap at lower Reynolds number regime to the spherical-cap bubble at higher Reynolds numbers $\left(50<\mathrm{Re}^{*}<100\right)$. As the bond number increases further $\left(100<\mathrm{Bo}^{*}<200\right)$, skirt bubbles are formed at higher Reynolds numbers $\left(20<\operatorname{Re}^{*}<100\right)$. When the Bond number is increased to the range of $100<\mathrm{Bo}^{*}<200$ and Reynolds number to the range $100<\mathrm{Re}^{*}<200$, toroidal bubbles are observed. From the simulation results, presented here, it can be concluded the current modelling method is robust enough to reasonably predict the various bubble shapes under wider flow regimes.

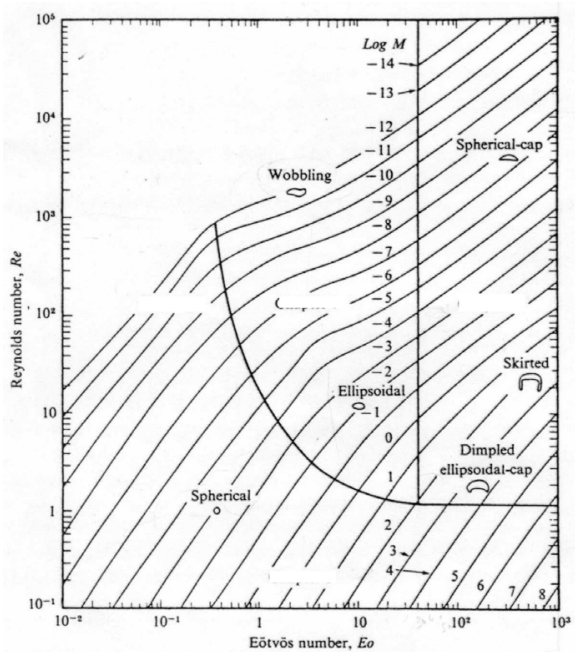

Figure 1: $\quad$ Bubble shapes under difference flow regimes (extracted from [8]).

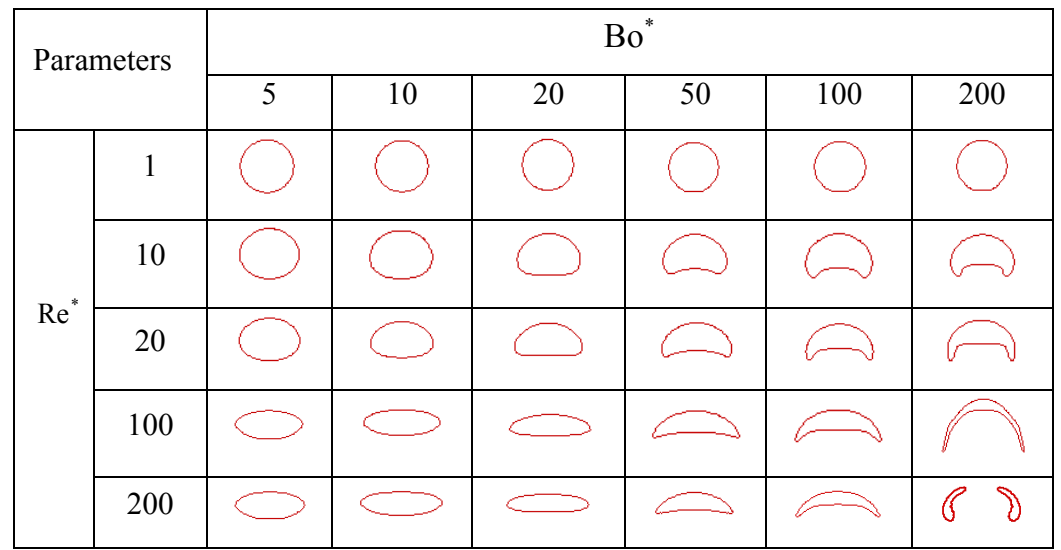

Figure 2: The predicted bubble shapes under various Reynolds and Bond numbers. 


\begin{tabular}{|c|c|c|c|c|}
\hline \multirow{2}{*}{ Test Case } & \multicolumn{2}{|c|}{ Experiments } & \multicolumn{2}{|c|}{ Simulations } \\
\hline & Test condition & $\begin{array}{l}\text { Observed bubble } \\
\text { terminal shape }\end{array}$ & $\begin{array}{l}\text { Predicted bubble } \\
\text { Terminal Shape }\end{array}$ & $\begin{array}{l}\text { Modelling } \\
\text { conditions }\end{array}$ \\
\hline TS1 & $\begin{array}{l}E=8.67 \\
M=711 \\
\mathrm{Re}=0.078\end{array}$ & & & $\begin{array}{l}\mathrm{Bo}=8.67 \\
\mathrm{Re}^{*}=0.979 \\
\mathrm{U}^{*}=0.069 \\
\mathrm{Re}, \mathrm{c}=0.0675\end{array}$ \\
\hline TS2 & $\begin{array}{l}\mathrm{E}=32.2 \\
\mathrm{M}=8.2 \times 10^{-4} \\
\mathrm{Re}=55.3\end{array}$ & & & $\begin{array}{l}\mathrm{Bo}=32.2 \\
\mathrm{Re}^{*}=79.88 \\
\mathrm{U}^{*}=0.663 \\
\mathrm{Re}, \mathrm{c}=52.96\end{array}$ \\
\hline TS3 & $\begin{array}{l}E=243 \\
M=266 \\
R e=7.77\end{array}$ & & & $\begin{array}{l}\mathrm{Bo}=243 \\
\mathrm{Re}^{*}=15.24 \\
\mathrm{U}^{*}=0.551 \\
\mathrm{Re}, \mathrm{c}=8.39\end{array}$ \\
\hline TS4 & $\begin{array}{l}\mathrm{E}=115 \\
\mathrm{M}=4.63 \times 10^{-3} \\
\mathrm{Re}=94.0\end{array}$ & & 1 & $\begin{array}{l}\mathrm{Bo}=115 \\
\mathrm{Re}^{*}=134.6 \\
\mathrm{U}^{*}=0.659 \\
\mathrm{Re}, \mathrm{c}=88.70\end{array}$ \\
\hline TS5 & $\begin{array}{l}E=641 \\
M=43.1 \\
R e=30.3\end{array}$ & & & $\begin{array}{l}\mathrm{Bo}=641 \\
\operatorname{Re}^{*}=49.72 \\
\mathrm{U}^{*}=0.602 \\
\mathrm{Re}, \mathrm{c}=29.93\end{array}$ \\
\hline
\end{tabular}

Figure 3: Comparison of terminal bubble shapes and rising speeds predicted by simulation and observed experiments.

\subsection{Comparison of terminal bubble shapes}

Detail comparison of the bubble shapes and terminal velocity is also necessary to understand the accuracy of the numerical predictions. Figure 3 compares the terminal bubble shapes and rising speeds obtained from experiments and simulations under various flow conditions. Typical bubble shapes (spherical, ellipsoid, ellipsoid-cap, spherical-cap and skirt bubbles) formed in different regimes can be predicted accordingly through simulations. The similarity of bubble shapes predicted in the simulations and observed in experiments is quite reasonable. For example, the simulation results on predicted bubble shape presented in Figure 3 shows that the bubble base is dimples or indented at the intermediate Reynolds number $\left(10<\mathrm{Re}^{*}<100\right)$ and relatively high Bond number $\left(20<\mathrm{Bo}^{*}<50\right)$. The indentation is also clearly visible in bubble photographs shown in cases TS3 and TS4, where the upper indentation may be seen near the axis of bubbles. At the rim of bubble, the different refractive indices of the gas and the liquid prevent us from seeing how the indentation joins the outer surface of the bubble. If taken this effect into consideration, the simulation predicted terminal bubble shapes agree well with experimental observation for the most study cases. 
The terminal bubble velocity is another important indicator to quantitatively evaluate the difference between the experimental and simulations. Based on the parameters given in the experiments, we can derive out the dimensionless parameters $\left(\mathrm{Re}^{*}, \mathrm{Bo}^{*}, \rho_{l} / \rho_{b}, \mu_{l} / \mu_{b}\right)$ to perform simulations. The simulation can predict the terminal bubble rising velocity $\left(U^{*}\right)$. Actually, based on this dimensionless velocity, the dimensional terminal bubble velocity $U_{\infty}$ can be calculated as $U^{*} \cdot g D^{1 / 2}$, and the dimensional terminal bubble velocity based Reynolds number ( $R e, c)$ predicted in simulation can be calculated as $\operatorname{Re}, \mathrm{c}=\operatorname{Re}^{*} \cdot U^{*}$. The comparison of the Reynolds numbers for the experiment $(\mathrm{Re})$ and simulation cases $(\mathrm{Re}, \mathrm{c})$ is also shown in Figure 3. The results from simulation prediction agree with those of experiment with very well within $10 \%$ difference.

\begin{tabular}{|c|c|c|c|c|}
\hline \multirow{2}{*}{$\begin{array}{l}\text { Test } \\
\text { Case }\end{array}$} & \multicolumn{2}{|c|}{ Experiments } & \multicolumn{2}{|c|}{ Simulations } \\
\hline & Test conditions & $\begin{array}{l}\text { Observed terminal } \\
\text { bubble wake }\end{array}$ & $\begin{array}{l}\text { Predicted terminal } \\
\text { bubble wake }\end{array}$ & $\begin{array}{l}\text { Modelling } \\
\text { conditions }\end{array}$ \\
\hline TW1 & $\begin{array}{l}E=96.2 \\
M=0.962 \\
R e=18.2\end{array}$ & & & $\begin{array}{l}\mathrm{Bo} *=96.2 \\
\operatorname{Re}^{*}=31.0\end{array}$ \\
\hline TW2 & $\begin{array}{l}E=94.3 \\
M=4.85 \times 10^{-3} \\
\operatorname{Re}=77.9\end{array}$ & & & $\begin{array}{l}\mathrm{Bo}^{*}=94.3 \\
\mathrm{Re}^{*}=116.3\end{array}$ \\
\hline TW3 & $\begin{array}{l}E=114 \\
M=4.85 \times 10^{-3} \\
\operatorname{Re}=91.6\end{array}$ & & & $\begin{array}{l}\mathrm{Bo}^{*}=114 \\
\mathrm{Re}^{*}=134.11 \\
\operatorname{Re}, \mathrm{c}=\end{array}$ \\
\hline TW4 & $\begin{array}{l}E=292 \\
M=26.7 \\
R e=22.1\end{array}$ & & & $\begin{array}{l}\mathrm{Bo}^{*}=292 \\
\mathrm{Re}^{*}=31.07\end{array}$ \\
\hline
\end{tabular}

Figure 4: Comparison of bubble wake predicted by simulation and observed in experiments.

\subsection{Comparison of bubble wake flow patterns}

The existence of a closed toroidal wake has been observed in experiments [5] through the flow visualisation with the $\mathrm{H}_{2}$ tracers. Wake flow circulation patterns predicted by the simulation agree well with the observations in experimental as shown in Figure 4. The wake circulation within the bubble base 
indentation is clearly shown in the case TW1 by the photograph, where the trace track disappears behind the rim of the bubble. The similar wake circulation pattern is revealed in the simulation. Actually, the simulation show that a second wake circulation occurs just behind the bubble rim. This may be the reason that the bubble photo of TW1 has some bright spots at outside of bubble rim. The second circulation in the skirt bubble wake becomes much clear from the simulation. The photo for the skirt bubble wake (TW4) does have much large bright spot just underneath the bubble. As the bubble size increase (Reynolds number increases), the wake volume increase as well, the wake seems to be torn away from the bubble (TW2 and TW3). In this case, the second bubble wake circulation disappears, and the bubble base indentation becomes smaller.

Compared to the experimental studies [5] on the rising bubble shapes in liquid within different flow regimes, the bubble shapes predicted using the present modelling approach are in reasonable agreement. Most of the previous numerical studies on bubble rising in liquid are limited to certain regimes where the bubble shape deformation is minimal. For example, in the work of [1], the bubble rise was simulated in the regimes of low Reynolds or low Bond numbers. The present work extends the capability to simulate the bubble rise and deformation for a wider flow regime.

\section{Conclusions}

A front tracking method for modelling two-phase fluid systems has been examined, improved and validated for much wider flow regimes. The new algorithm adopted the treatment of the interface as finite thickness as proposed by Tryggvason et al. The fluid properties (density, viscosity and surface tension) were varied smoothly over the interface, and updated with the new interface position. The interface is advected using the front marker velocities that are interpolated from the velocity field in the fixed grid. The front mesh size is adapted to match the background mesh size due to it variation in front moving and deforming, and the front mesh position is corrected to conserve the front inner volume. The velocity field has been solved implicitly with the finite volume method over the fixed grid using the improved SIMPLE algorithm, which keeps the conservation of volume flux. The newly proposed algorithm is applied to simulate the rise of single bubble in a viscous liquid. The bubble shapes and velocity in a wider flow regime are studied as a function of the nondimensional parameters such as Reynolds number, Bond number, density ratio and viscosity ratio. The comparison of simulations with the available experiments shows satisfactory agreements in terminal bubble shape, velocity and wake flow pattern.

\section{References}

[1] Unverdi, S. O. \& Tryggvason, G. A front-tracking method for viscous, incompressible, multi-fluid flows. J. Comput. Phys. 100, 25-37, 1992. 
[2] Bunner, B. \& Tryggvason, G. Dynamics of homogenous bubbly flows. Part 1. Rise velocity and microstructure of the bubbles. J. Fluid Mech. 466, 17-52, 2002.

[3] Chen, L., Garimella, S. V., Reizes, J. A. \& Leonardi, E., The development of a bubble rising in a viscous liquid. J. of Fluid Mech. 387, 61-96, 1999.

[4] Ohta, M., Imura, T., Yoshida, Y. \& Sussman M. A computational study of the effect of initial bubble conditions on the motion of a gas bubble rising in viscous liquids. Intl. J. of Multiphase flow 31, 223-237, 2005.

[5] Bhaga, D. \& Weber, M. E. Bubbles in viscous liquid: shapes, wakes and velocities. J. Fluid Mech. 105, 61-85, 1981.

[6] Peskin, C.S. \& Printz, B.F. Improved volume conservation in the computation of flows with immersed boundaries, J. Comput. Phys. 105, 33-46, 1993.

[7] Patankar, S.V. Numerical heat transfer and fluid flow. Hemisphere, 1980.

[8] Clift, R., Grace, J. R. \& Weber, M. E. Bubbles, Drops, and Particles. Academic Press, 1978. 